\title{
Fingerprint Segmentation Algorithms: A Literature Review
}

\author{
Rohan Nimkar \\ Dept. of Electronics and Telecommunication \\ Jabalpur Engineering College, \\ Jabalpur (M.P.)
}

\author{
Agya Mishra, Ph.D \\ Assistant Professor \\ Dept. of Electronics and Telecommunication \\ Jabalpur Engineering College, Jabalpur (M.P.)
}

\begin{abstract}
Fingerprint image segmentation is part of pre-processing in fingerprint image recognition system. It has a critical effect to the fingerprint image recognition system. This paper review the different algorithms particularly used for fingerprint segmentation. Adaptive Total Variation Model, Directional Total Variation Model, method based on combination of ridge orientation and frequency features, method based on orientation field information combined with statistical characteristics of gray, Ridge Template Correlation, method using three pixel features(being the coherence, the mean and the variance) are discussed and compared based on their performance and concluded that Adaptive Total Variation Model and Directional Total Variation Model provides better results, so it will be good for the field of researches.
\end{abstract}

\section{General Terms}

Image recognition, fingerprint image preprocessing, algorithms of fingerprint segmentation.

\section{Keywords}

Image recognition, fingerprint segmentation, fingerprint image pre-processing, total variation, ridge orientation.

\section{INTRODUCTION}

A biometric is any physical or behavioral trait that can be used to identify a person. The most common biometric traits are face, speech, fingerprint, and iris. Fingerprints are one of the most widely used traits due to their universality, distinctiveness and performance. From the creation of the first Automated Fingerprint Identification System (AFIS), a tremendous amount of effort and progress has been made to match sample prints accurately and consistently with large fingerprint databases. An essential step towards achieving accurate identification is an accurate segmentation.

On the basis of collection procedure, fingerprint images can generally be classified into three categories, namely, rolled, plain and latent [2]. Rolled fingerprints are obtained from rolling the finger from one side to the other in order to capture all ridge details of the fingerprint. Plain fingerprints images are acquired by pressing the fingertip onto a flat surface. Since rolled and plain prints are obtained in an attended mode, so they are usually of good visual quality and contain sufficient information for reliable matching. However, latent fingerprints are usually collected from crime scenes, in which the print is lifted from object surfaces that were inadvertently touched or handled. The matching between latent and rolled/plain fingerprints plays a crucial role in identifying suspects by law enforcement agencies.
Fingerprint segmentation is part of pre-processing in fingerprint image recognition system and it refers to the process of decomposing a fingerprint image into two disjoint regions: foreground and background. The foreground, also called the region of interest (ROI), consists of the desired fingerprints while the background contains noisy and irrelevant contents that will be discarded in the following processing steps. Accurate fingerprint segmentation is critical, as it affects the accurate extraction of minutiae and singular points, which are key features for fingerprint matching and hence also for fingerprint image recognition system. When feature extraction algorithms are applied on a fingerprint image without segmentation, lots of false features may be extracted due to the presence of noisy background, and eventually leading to matching errors in the later stage. Therefore, the goal of fingerprint segmentation is to discard the background, reduce the number of false features, and thus improve the matching accuracy. So considering the importance of segmentation for accurate matching of fingerprint, we have discuss about a segmentation algorithm in our work.

A number of fingerprint segmentation methods are known from literature, which can be roughly divided into block-wise methods and pixel-wise methods. Block-wise methods first partition a fingerprint image into non overlapping blocks of the same size, and then classify the blocks into foreground and background based on the extracted block-wise features. Pixelwise methods classify pixels through the analysis of pixelwise features. The commonly used features in fingerprint segmentation include gray-level features, orientation features, frequency domain features, and so forth. The effective segmentation algorithms such as Adaptive Total Variation Model, Directional Total Variation Model, method based on combination of ridge orientation and frequency features, method based on orientation field information combined with statistical characteristics of gray, Ridge Template Correlation, method using three pixel features (being the coherence, the mean and the variance) are discussed and compared based on their performance in this paper.

\section{EXISTING SEGMENTATION ALGORITHMS}

Several approaches to fingerprint image segmentation are known from literature, such as Adaptive Total Variation Model [1], Directional Total Variation Model [2], method based on combination of ridge orientation and frequency features [3], method based on orientation field information combined with statistical characteristics of gray [4], Ridge Template Correlation [5], method using three pixel features, being the coherence, the mean and the variance [6]. 

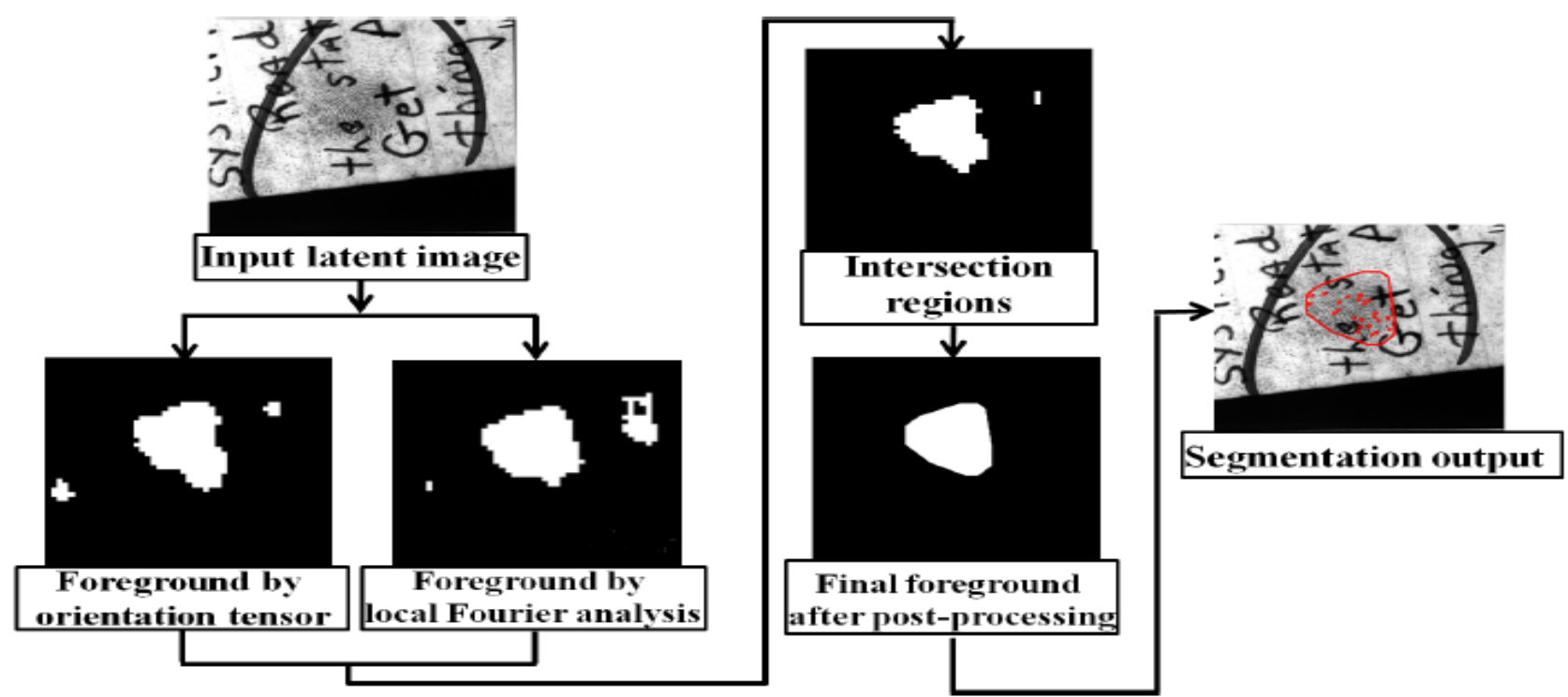

Fig 1: A flowchart of the Method based on combination of ridge orientation and frequency features. [3]

\subsection{Adaptive Total Variation Model}

Adaptive Total Variation Model [1] is based on TV-L1 model.TV-L1 model decomposes an input image, ' $f$ ', into two signal layers, cartoon ' $u$ ', which consists of the piecewisesmooth component in f, i.e. contains both structured noise and small-scale structures, and texture ' $\mathrm{v}$ ', which contains the oscillatory or textured component in $\mathrm{f}$, i.e. consists of latent fingerprints and a small amount of noise. In Adaptive TV-L1 model the weight coefficient $\lambda$ (of TV-L1 model) of the fidelity term can be adaptively adjust depending on the background noise level, which is estimated via TV-based texture analysis.

The decomposition:

$$
\mathrm{f}=\mathrm{u}+\mathrm{v}
$$
is obtained by solving the following variational problem:

$$
\min _{\mathrm{u}} \int|\nabla \mathrm{u}|+\int \lambda(\mathrm{x})|\mathrm{u}-\mathrm{f}| \mathrm{dx}
$$

where $\mathrm{f}, \mathrm{u}$ and $\mathrm{v}$ are functions of image gray-scale intensity values in $\mathrm{R}^{2}, \nabla \mathrm{u}$ is the gradient value of $\mathrm{u}$ and $\lambda(\mathrm{x})$ is a spatially varying parameter. $\int|\nabla \mathrm{u}|$ and $|\mathrm{u}-\mathrm{f}|$ the total variation of $\mathrm{u}$ and the fidelity term, respectively. In the fingerprint region, the $\lambda(\mathrm{x})$ value should be relatively small, since low fidelity ensures the smoothness of $u$ and, thus, more textures could be extracted in v. In regions with structured noise, fidelity becomes important since a large $\lambda(x)$ value ensures all noise components to be filtered out from texture $\mathrm{v}$.

This algorithm provides very satisfactory segmentation results but for the worst (author says ugly) latent prints the result is not accurate.

\subsection{Directional Total Variation Model}

The Directional Total Variation (DTV) model [2], based on TV-L2 model, is described to achieve efficient latent fingerprint detection and segmentation.TV-L2 model decomposes an images in similar way as TV-L1 model described above. In addition to TV-L2 model, the DTV model uses orientation vector $\vec{a}$ that controls the signal captured in the texture output ' $v$ '. The decomposition (1) is obtained by solving the following variational problem

$$
\min _{\mathrm{u}} \int|\nabla \mathrm{u} \cdot \overrightarrow{\mathrm{a}}(\mathrm{x})| \mathrm{dx}+\frac{\lambda}{2}\|\mathrm{f}-\mathrm{u}\|^{2}
$$

where $\vec{a}(x)$ is spatially varying orientation vector adjusted to local texture orientation.

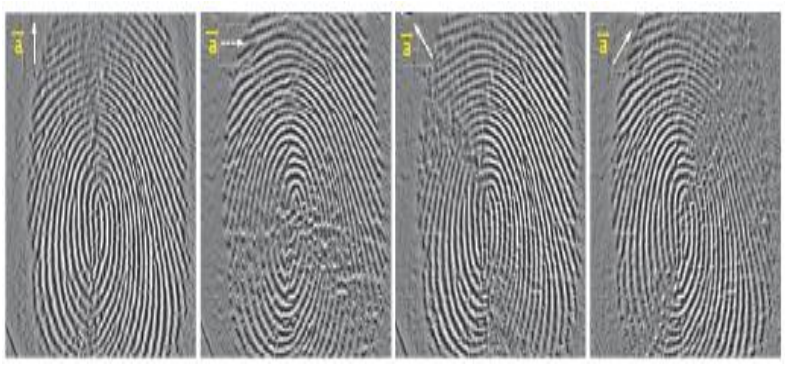

Fig 2: Texture output $\mathbf{v}$ for $\vec{a}$ in four different direction [2]

By tuning $\vec{a}$ to a specific direction, we are mainly interested in minimizing the total variation of ' $u$ ' along that direction, while allowing the existence of total variation of ' $u$ ' along other directions. As a result, textures along the corresponding direction will be fully captured by ' $v$ ' and textures of other directions will be weakened in ' $v$ '. Above figure, from [2], illustrates the effect of $\vec{a}$ on the output of 'v'.This algorithm performs well on images with oriented textures.

\subsection{Method based on combination of Ridge Orientation and frequency features}

In [3], the algorithm utilizes both ridge orientation and frequency features. The orientation tensor approach is used to extract the symmetric patterns of a fingerprint as well as to remove the structured noise in background. Local Fourier analysis method is used to estimate the local frequency in the latent fingerprint image and locate fingerprint region by considering valid frequency regions. Candidate fingerprint (foreground) regions are obtained for each feature (orientation and frequency) and then an intersection of these regions is used to localize the latent fingerprint region. 
As compared to manual segmentation of images, it provides satisfactory results. A flowchart of this method is shown in figure 1.

\subsection{Method based on orientation field information combined with statistical characteristics of gray}

Combined method segmentation is proposed in [4] which use the orientation field information combined with statistical characteristics of gray. The gray level statistical features (such as mean value, variance) are used for background segmentation of a large area and small noise area's segmentation. The algorithm conducts secondary segmentation (orientation field information) in blocks and finally, obtains the block orientation.

For saving processing time the algorithm is well but limited by the case of images that are too wet or too dry.

\subsection{Three Pixel features Method}

The method in [6] uses three pixel features, being the coherence, the mean and the variance. An optimal linear classifier is trained for the classification per pixel, while morphology is applied as post processing to obtain compact clusters and to reduce the number of classification errors.

\subsubsection{Pixel Feature Extraction}

The first step is the selection of pixel feature on which the segmentation will be based.

The first pixel feature is coherence. The coherence gives a measure how well the gradients are pointing in the same direction. Since a fingerprint mainly consists of parallel line structures, the coherence will be considerably higher in the foreground than in the background. In a window $W$ around a pixel, the coherence is defined as:

$$
\operatorname{Coh}=\frac{\left|\sum_{\mathrm{w}}\left(\mathrm{G}_{\mathrm{s}, \mathrm{x}} \mathrm{G}_{\mathrm{s}, \mathrm{y}}\right)\right|}{\sum_{\mathrm{w}}\left|\left(\mathrm{G}_{\mathrm{s}, \mathrm{x}}, \mathrm{G}_{\mathrm{s}, \mathrm{y}}\right)\right|}=\frac{\sqrt{\left(\mathrm{G}_{\mathrm{xx}}-\mathrm{G}_{\mathrm{yy}}\right)^{2}+4 \mathrm{G}_{\mathrm{xy}}^{2}}}{\mathrm{G}_{\mathrm{xx}}+\mathrm{G}_{\mathrm{yy}}}
$$

where (Gs, $\mathrm{x}, \mathrm{Gs}, \mathrm{y})$ is the squared gradient, $\mathrm{Gxx}=\sum_{\mathrm{W}} \mathrm{G}_{\mathrm{x}}^{2}$, Gyy $=\sum_{w} G_{y}^{2}$, Gxy $=\sum_{w} G_{x} G_{y}$ and $(G x, G y)$ is the local gradient.

The average gray value is the second pixel feature. The mean gray value in the foreground is general lower, i.e. darker gray, than it is in the background. Using I as the intensity of the image, the local mean for each pixel is given by:

$$
\text { Mean }=\sum_{\mathrm{W}} \mathrm{I}
$$

The variance is the third pixel feature that can be used. In general, the variance of the ridge-valley structures in the foreground is higher than the variance of the noise in the background. The variance for each pixel given by:

$$
\operatorname{Var}=\sum_{\mathrm{W}}(\mathrm{I}-\mathrm{Mean})^{2}
$$

\subsubsection{Classification}

It uses a linear classifier, which tests a linear combination of the features, given by:

$$
\mathrm{v}=\mathrm{w}^{\mathrm{T}} \mathrm{x}=\mathrm{w}_{0} \text { Coh }+\mathrm{w}_{1} \text { Mean }+\mathrm{w}_{2} \text { Var }+\mathrm{w}_{3}
$$

Where $\mathrm{v}$ is the value to be tested, $\mathrm{w}=\left[\begin{array}{llll}\mathrm{w}_{0} & \mathrm{w}_{1} & \mathrm{w}_{2} & \mathrm{w}_{3}\end{array}\right]^{\mathrm{T}}$ is the weight vector and $\mathrm{x}=[\text { Coh Mean Var } 1]^{\mathrm{T}}$.

\subsubsection{Post Processing}

Morphology is applied to the classification estimate. It reduces the number of false classifications. First, small clusters that are incorrectly assigned to the foreground are removed. Next, small clusters that are incorrectly assigned to the background are removed.

This algorithm is the simplest one to be adopted but lengthy in implementation. More information on this method can be seen in [6].

\subsection{Ridge Template Correlation method}

The approach in [5] models an "ideal" ridge section with a sinusoid that is adjusted to local contrast, frequency, and direction. The image is sectioned into local blocks that are compared to the generated template, and then a 'goodness of fit" score is computed which are used to assign quality levels to the block. A consequence of assigning quality levels is that the background is identified, using the criterion that background regions will exhibit a lack of positive correlation with the template structure.

This algorithm provides satisfactory result under certain limitation (specified in Table 1).Workflow of this method is represented in figure 3 .

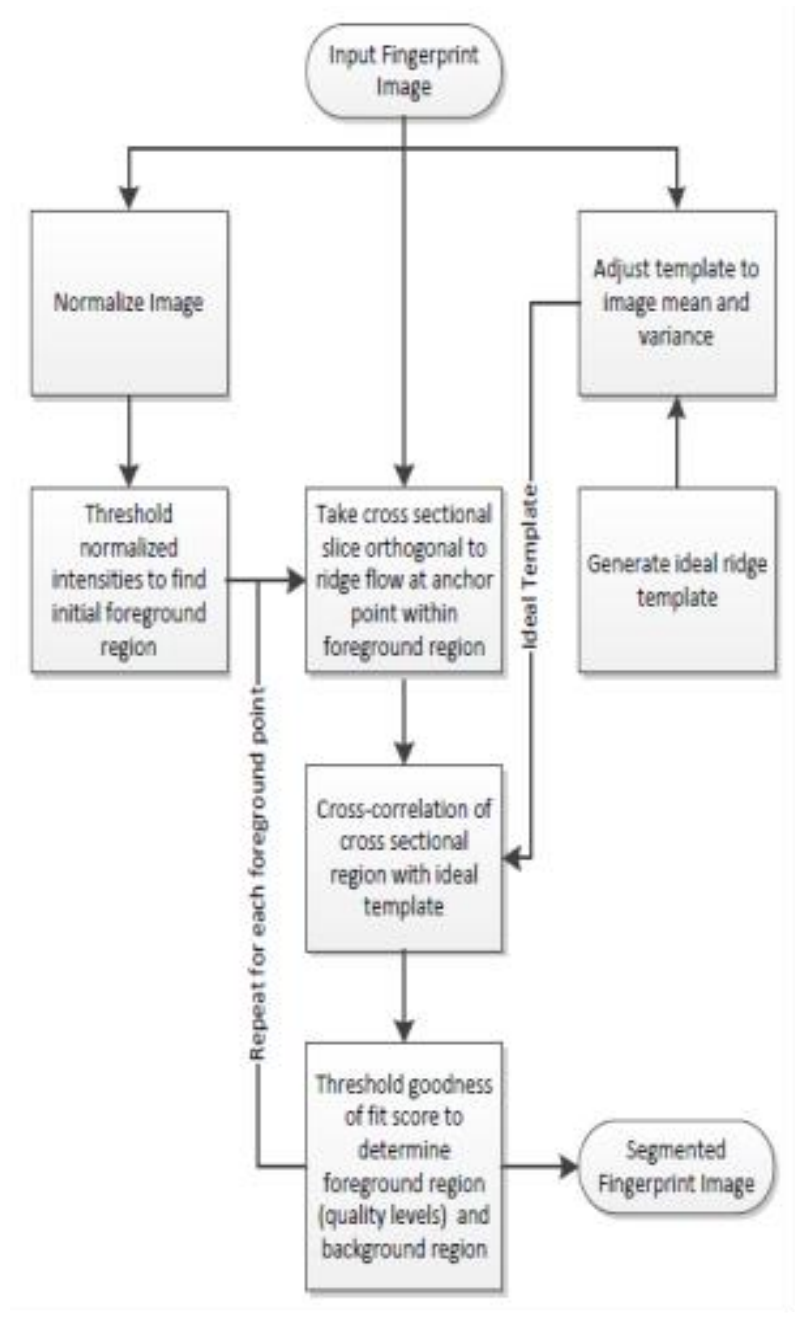

Fig 3: Work Flow for Ridge Template Correlation method. [5] 


\section{COMPARISON OF DIFFERENT}

\section{ALGORITHMS}

Different segmentation algorithms discuss above are compared in table 1 under some features.

Table 1.Comparison of Published Fingerprint Segmentation Algorithms

\begin{tabular}{|c|c|c|c|c|c|c|}
\hline References & $\begin{array}{l}\text { Segmentation } \\
\text { Algorithm }\end{array}$ & $\begin{array}{l}\text { Measuring } \\
\text { Parameters }\end{array}$ & $\begin{array}{l}\text { Computational } \\
\text { Complexities }\end{array}$ & Limitations & Advantages & Applications \\
\hline [1] & $\begin{array}{c}\text { Adaptive Total } \\
\text { Variation Model. }\end{array}$ & $\begin{array}{c}\lambda=\text { Fidelity } \\
\text { Weight } \\
\text { Coefficient and } \\
\text { features such as } \\
\text { mean, variance } \\
\text { and coherence }\end{array}$ & $\begin{array}{c}\text { In selecting } \\
\text { different values } \\
\text { of } \lambda \text { in different } \\
\text { fingerprint } \\
\text { image region. }\end{array}$ & $\begin{array}{c}\text { Accurate } \\
\text { performance } \\
\text { is not } \\
\text { achieved for } \\
\text { worst (author } \\
\text { says ugly) } \\
\text { latent } \\
\text { fingerprints }\end{array}$ & $\begin{array}{l}\text { Provides very } \\
\text { satisfactory } \\
\text { segmentation } \\
\text { results. }\end{array}$ & $\begin{array}{l}\text { (i)Fingerprint } \\
\text { Segmentation. } \\
\text { (ii)Image } \\
\text { Decomposition }\end{array}$ \\
\hline [2] & $\begin{array}{l}\text { Directional Total } \\
\text { Variation Model. }\end{array}$ & $\begin{array}{c}\overrightarrow{\mathrm{a}(\mathrm{x})}=\text { spatially } \\
\text { varying } \\
\text { orientation vector } \\
\text { and use variance } \\
\text { feature. }\end{array}$ & $\begin{array}{c}\text { In keeping } \overrightarrow{\mathrm{a}(\mathrm{x})} \\
\text { spatially varying } \\
\text { and well aligned } \\
\text { with local } \\
\text { fingerprint ridge } \\
\text { orientation }\end{array}$ & - & $\begin{array}{c}\text { Good } \\
\text { performance } \\
\text { as compared } \\
\text { to [1]'s } \\
\text { method. }\end{array}$ & $\begin{array}{c}\text { Decomposition } \\
\text { of images with } \\
\text { oriented textures. }\end{array}$ \\
\hline [3] & $\begin{array}{l}\text { Method based on } \\
\text { combination of ridge } \\
\text { orientation and } \\
\text { frequency features. }\end{array}$ & $\begin{array}{l}\text { Ridge frequency } \\
\text { or Ridge density } \\
\text { and mean value }\end{array}$ & $\begin{array}{l}\text { Orientation and } \\
\text { frequency } \\
\text { features are } \\
\text { determined for } \\
\text { each print } \\
\text { separately and } \\
\text { then their } \\
\text { intersection is } \\
\text { taken. }\end{array}$ & $\begin{array}{l}\text { Need a robust } \\
\text { confidence } \\
\text { measure for } \\
\text { segmentation } \\
\quad \text { output }\end{array}$ & $\begin{array}{l}\text { Satisfactory } \\
\text { results as far } \\
\text { as visual } \\
\text { inspection is } \\
\text { concerned. }\end{array}$ & $\begin{array}{l}\text { Detection and } \\
\text { Segmentation of } \\
\text { Latent } \\
\text { Fingerprint }\end{array}$ \\
\hline [4] & $\begin{array}{l}\text { Combined Method. } \\
\text { Orientation field } \\
\text { information } \\
\text { combined with } \\
\text { statistical } \\
\text { characteristics of } \\
\text { gray. }\end{array}$ & $\begin{array}{l}\text { Mean gray value } \\
\text { and variance. }\end{array}$ & - & $\begin{array}{l}\text { Not so good } \\
\text { for image that } \\
\text { is too wet or } \\
\text { too dry. }\end{array}$ & $\begin{array}{l}\text { (i)Improves } \\
\text { accuracy } \\
\text { (ii)Saves } \\
\text { processing } \\
\text { time }\end{array}$ & $\begin{array}{c}\text { Fingerprint } \\
\text { Image } \\
\text { Segmentation }\end{array}$ \\
\hline [5] & $\begin{array}{c}\text { Ridge Template } \\
\text { Correlation. }\end{array}$ & $\begin{array}{l}\text { Image mean and } \\
\text { variance }\end{array}$ & $\begin{array}{l}\text { Lengthy } \\
\text { algorithm need } \\
\text { to be followed } \\
\text { in order to } \\
\text { achieve } \\
\text { effective } \\
\text { segmentation }\end{array}$ & $\begin{array}{l}\text { When } \\
\text { fingerprint is } \\
\text { large and } \\
\text { missed } \\
\text { minutiae is } \\
\text { high, the } \\
\text { segmentation } \\
\text { incorrectly } \\
\text { labels the } \\
\text { background as } \\
\text { foreground }\end{array}$ & $\begin{array}{c}\text { Reduces the } \\
\text { average } \\
\text { detected } \\
\text { fingerprint } \\
\text { area from } \\
60.7 \% \text { of the } \\
\text { total image to } \\
33.6 \% \text {. }\end{array}$ & $\begin{array}{c}\text { Fingerprint } \\
\text { Segmentation }\end{array}$ \\
\hline [6] & $\begin{array}{c}\text { Three pixel features, } \\
\text { the coherence, the } \\
\text { mean and the } \\
\text { variance. }\end{array}$ & $\begin{array}{c}\text { Coherence, Mean } \\
\text { and Variance. }\end{array}$ & $\begin{array}{l}\text { Post processing } \\
\text { of segmented } \\
\text { fingerprint } \\
\text { image is } \\
\text { necessarily } \\
\text { required }\end{array}$ & $\begin{array}{l}6.8 \% \text { of the } \\
\text { pixels is } \\
\text { misclassified } \\
\text { (while the } \\
\text { post } \\
\text { processing } \\
\text { reduces this } \\
\text { ratio.) }\end{array}$ & $\begin{array}{l}\text { Accurate } \\
\text { high- } \\
\text { resolution } \\
\text { segmentation } \\
\text { results. }\end{array}$ & $\begin{array}{c}\text { Fingerprint } \\
\text { Segmentation. }\end{array}$ \\
\hline
\end{tabular}




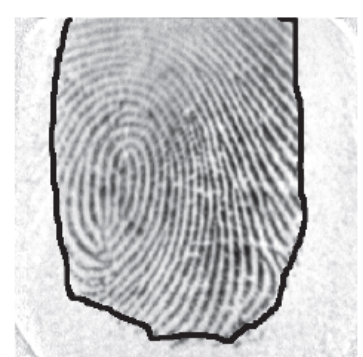

Plain Fingerprint

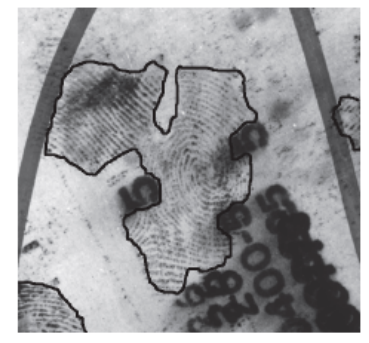

Latent Fingerprint

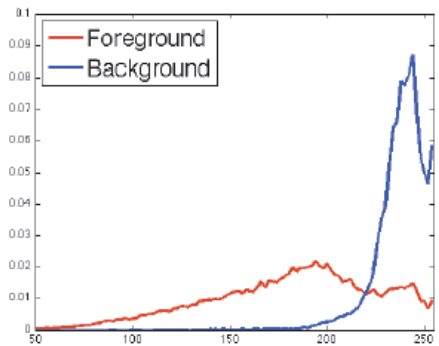

Mean

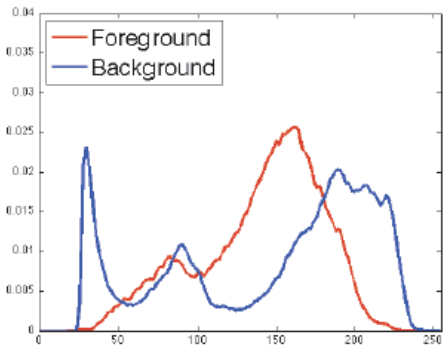

Mean

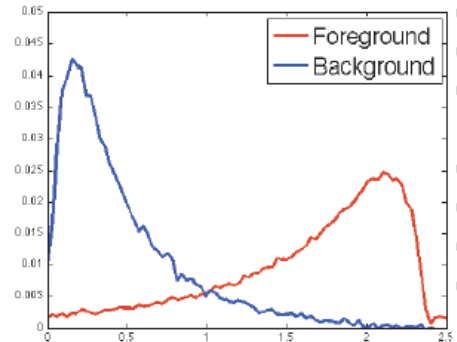

Variance

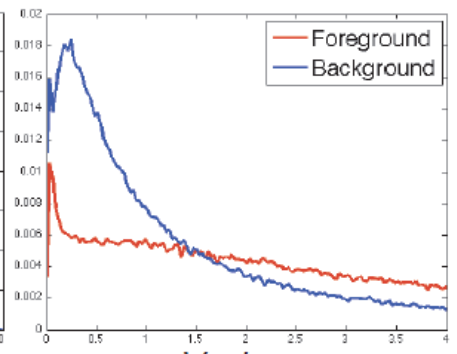

Variance

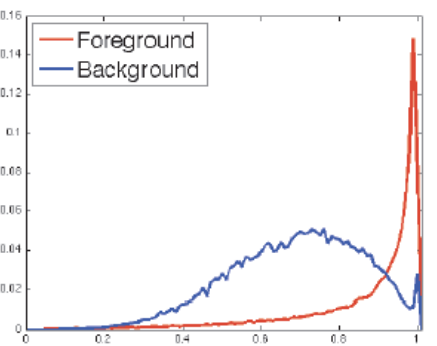

Coherence

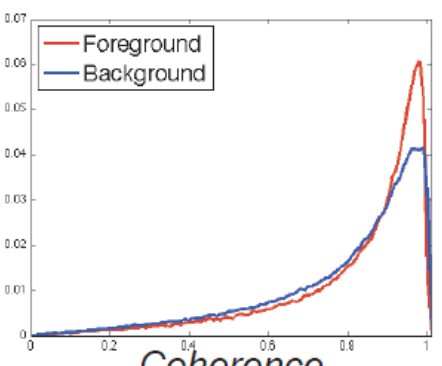

Cơherence

Fig 4: Comparison of distributions of the mean, variance and coherence features in the foreground and background areas of plain and latent fingerprints. [2]

All the discussed algorithms gives much accurate segmentation results as far as plain and rolled fingerprints are concerned, but for the case of latent fingerprints, segmentation results are not highly accurate. Accuracy of methods utilizing only pixels features for segmentation are highly limited by the quality of fingerprints obtained. Complexities in segmenting latent fingerprints as compared to plain/rolled fingerprints can better be understood by the distribution plot of three segmentation features; namely, the mean, variance and coherence, in Figure 4 [2] for plain and latent fingerprint image separately. As shown in the figure 4, the distributions of these features in foreground and background regions are well separated for plain fingerprints. In contrast, those of latent fingerprints have a significant overlap.

To improve results of segmentation of poor quality fingerprint images, other features such as ridge patterns, frequency features, orientation vectors etc. are combined with pixels features for decomposing the images. Adaptive Total Variation model and Directional Total Variation model overcome some drawbacks of poor quality images in its segmentation by implementing other features in addition to pixel features in its algorithm, providing much satisfactory results as compared to other algorithms or methods.

\section{CONCLUSION}

Current automated fingerprint identification systems have achieved high accuracy in matching fingerprints, segmentation still remains to be a challenging problem and requires much human intervention. In this paper, different segmentation algorithms are discussed, each having a different accuracy of segmenting fingerprint image. This paper concludes the critical review of fingerprint segmentation, with all the advantages, limitations and complexities of algorithms. Finally it concludes that, algorithms that performs better for poor fingerprint images and latent fingerprints are considerable, so Adaptive Total Variation Method [1] and Directional Total Variation Model [2] is good to implement.

\section{REFERENCES}

[1] Jiangyang Zhang, Rongjie Lai and C.C. Jay Kuo. 2012. Latent Fingerprint Segmentation with Adaptive Total Variation Model. IEEE.

[2] Jiangyang Zhang, Rongjie Lai and C.-C. Jay Kuo. 2012. Latent Fingerprint Detection and Segmentation with a Directional Total Variation Model. IEEE.

[3] Heeseung Choi, Maurilio Boaventura, Ines A.G. Boaventura and Anil K. Jain, Automatic Segmentation of Latent Fingerprints.

[4] Juntao Xue and Hongwei Li. 2012. Fingerprint image segmentation based on a combined method. IEEE.

[5] Nathan J. Short, Michael S. Hsiao, A. Lynn Abbott and Edward A. Fox. Latent Fingerprint Segmentation using Ridge Template Correlation.

[6] Asker M. Bazen and Sabih H. Gerez. 2001. Segmentation of Fingerprint Images, Workshop on Circuits, Systems and Signal Processing, Veldhoven. The Netherlands. 